\title{
Variable Genomic and Metabolomic Responses to Varying Doses of Vitamin D Supplementation
}

\author{
ARASH SHIRVANI ${ }^{1}$, TYLER AREK KALAJIAN ${ }^{1}$, ANJELI SONG ${ }^{1}$, RACHEL ALLEN ${ }^{1}$, \\ NIPITH CHAROENNGAM ${ }^{1}$, RICHARD LEWANCZUK ${ }^{2}$ and MICHAEL F. HOLICK ${ }^{1}$ \\ ${ }^{1}$ Boston Medical Campus, Section Endocrinology, Diabetes, Nutrition and Weight Management, \\ Department of Medicine, Vitamin D, Skin, and Bone Research Laboratory, \\ Boston University School of Medicine, Boston, MA, U.S.A.; \\ ${ }^{2}$ Division of Endocrinology and Metabolism, Department of Medicine, \\ University of Alberta and Alberta Health Services, Edmonton, AB, Canada
}

\begin{abstract}
Background/Aim: To assess the impact of vitamin $D$ supplementation on genomic and metabolomic profiles and relate them to the individual's responsiveness to varying doses of vitamin $D_{3}$. Patients and Methods: Healthy adults were given either 600,4000 or 10,000 IUs vitamin $D_{3} /$ day for 6 months. Circulating parathyroid hormone (PTH), 25 hydroxyvitamin D [25(OH)D], calcium, peripheral white blood cells broad gene expression and urine and serum metabolomic profiles were evaluated. Results: There was a dose-dependent effect of vitamin D supplementation on serum 25(OH)D, PTH and broad gene expression. Serum calcium levels remained normal for all study subjects and no untoward toxicity was observed. The metabolomic profiles were related to the genomic expression analysis. There were significant inter-individual effects on gene expression and metabolomic profile in response to the same dose of vitamin $D_{3}$ supplementation, despite similar changes in $25(\mathrm{OH}) \mathrm{D}$ and PTH concentrations. Conclusion: These results may help explain the variability observed in clinical trials regarding vitamin D's non-calcemic health benefits.
\end{abstract}

It is generally accepted that vitamin D, the sunshine vitamin, is essential for the development and maintenance of bone health throughout life (1-4). The non-skeletal health benefits of vitamin $\mathrm{D}$ are supported by the observations that the vitamin $\mathrm{D}$ receptor is expressed in several types of tissues

Correspondence to: Michael F. Holick, Ph.D., MD, Boston University School of Medicine, Vitamin D, Skin, and Bone Research Laboratory, 85 E Newton St. M-1013, Boston, MA, 02118, U.S.A. Tel: +1 6173586139, e-mail: mfholick@bu.edu

Key Words: Vitamin D, supplementation, metabolomics, genomics, broad gene expression, metabolomic profile, individual response, personalized medicine, 25-hydroxyvitamin D.
(1-4). A multitude of association studies and meta-analyses have demonstrated the potential benefits of vitamin D on longevity and reducing risk for cardio-metabolic disorders, several types of cancers, autoimmune disorders, all-cause mortality and other acute and chronic illnesses (1-3). However, the health benefit of vitamin D supplementation has been questioned based on recent clinical trials that not only challenged the concept of non-skeletal health benefits of vitamin $\mathrm{D}$, but also suggested a potential detrimental effect of increased vitamin D supplementation on bone health (4). Furthermore, some studies have reported that improvement in vitamin D status can improve bone mineral density and reduce risk of fracture while others have not seen any benefit (1-3).

Different interpretation of results from observational studies with vitamin D led to some dispute in the field on the desired optimal vitamin D status i.e. serum 25-hydroxyvitamin D $[25(\mathrm{OH}) \mathrm{D}]$ and also suggest that there may be individual sensitivity and responsiveness to vitamin $\mathrm{D}_{3}$.

Two recent studies (VitDmet and VitDbol trials) have indicated that there were individual differences in response to supplementation with vitamin $\mathrm{D}_{3}(5,6)$. They concluded that the variable response to vitamin $\mathrm{D}_{3}$ made be explained by epigenetic and genetic individual differences $(5,6)$. Other possibilities include an individual's ability to convert vitamin $\mathrm{D}$ to its active metabolites and the interaction of 1,25 dihydroxyvitamin $\mathrm{D}_{3}\left[1,25(\mathrm{OH})_{2} \mathrm{D}_{3}\right]$ with its receptors and gene response elements (5-7).

The concept is based on the fact that $1 \alpha, 25(\mathrm{OH})_{2} \mathrm{D}_{3}$, serving as the transcription factor for the vitamin D receptor and thus has a direct effect on the epigenome and transcriptome of many human tissues and cell types (5-7). Individuals can be categorized as robust, weak and low or non-responders to vitamin $\mathrm{D}$ by measuring vitamin $\mathrm{D}$ sensitive molecular parameters, such as changes in the epigenetic status and the respective transcription of genes of 
blood immune cells. In addition, responsiveness can also be related to the changes and amounts of proteins or metabolites in the urine or serum (5).

Intracellularly, the interaction of $1,25(\mathrm{OH}) \mathrm{D}$ with its receptor is thought to result in some kind of physicochemical alterations, leading to its activation after complexing with retinoid acid $\mathrm{X}$ receptor. This heterodimer binds to the vitamin $\mathrm{D}$ response element of genes unlocking their genetic information (5-7). Our recent study has supported the concept of genomic responsiveness to vitamin D. We have shown that in subjects with a lower genomic response to vitamin $\mathrm{D}_{3}$ supplementation, there was a response in $\sim 2-5 \%$ of the genome while in more responsive subjects $>5 \%$ of the genome responded to vitamin $\mathrm{D}_{3}(8)$.

Some specific metabolites are known to be involved in this process of vitamin $\mathrm{D}$ dependent genomic activation. The experimental studies have shown that polyamines activate the vitamin D receptor in vitro and it is possible that they are related to the regulation of vitamin $\mathrm{D}$ action (9). The enhancement of spermidine $\mathrm{N}$-acetyltransferase activity by 1,25-dihydroxyvitamin $\mathrm{D}$ is observed not only in the classical target tissues but also in the newly recognized target tissues of vitamin D (10). Some of these target tissues do not appear to be involved in the regulation of mineral metabolism. Indeed, metabolomic studies on blood and urine from subjects supplemented with calcium and vitamin D revealed different metabolic profiles (11). Thus, it seems that there is a close interaction between vitamin D status and metabolic profiles that may explain the individual's response to vitamin D. Less attention has been given to the more basic fundamental concepts related to inter-individual differences in vitamin D optimization (12).

The aim of this randomized controlled double-blind clinical trial was to assess the impact of vitamin D supplementation on metabolomic profiles by relating them to the individual's responsiveness to varying doses of vitamin $\mathrm{D}_{3}$.

\section{Patients and Methods}

The protocol of this metabolomic study was approved by the Institutional Review Board (IRB) of Boston University Medical Campus (H-35506). This study is registered as a clinical trial at the ClinicalTrials.gov (NCT02856776; date of registration 05/08/2016) (8). All participants read and signed the written informed consent.

A deidentified bottle that contained 60 capsules of vitamin $\mathrm{D}_{3}$ was given to each participant that was formulated with one of the following doses of vitamin $\mathrm{D}_{3}$, $600 \mathrm{IU} /$ day, $4,000 \mathrm{IU} /$ day or 10,000 IU/day. All vitamin $\mathrm{D}_{3}$ supplements were provided by Solgar Inc (Leonia, NJ, USA) and the contents evaluated by HPLC as previously described (7) and were found to contain concentrations within $10 \%$ of their specified content. The bottles were returned at each visit when the vitamin $\mathrm{D}_{3}$ capsules were counted to track compliance.

The participants were randomized by a computer-generated randomization program into one of the three study groups.
Recruitment. Healthy young adult males and non-pregnant females were prescreened for serum concentrations of $25(\mathrm{OH}) \mathrm{D}$ deemed insufficient (below $30 \mathrm{ng} / \mathrm{ml}$ ). Inclusion criteria included healthy young black and white adults with a $\mathrm{BMI}<30 \mathrm{~kg} / \mathrm{m}^{2}$ without disorders or medications affecting vitamin $\mathrm{D}$ metabolism. To reduce the effect daylight on 25(OH)D level recruitment started in October and supplementation was completed by March. All subjects signed an informed consent that was accepted by the IRB of Boston University Medical Campus (8).

The exclusion criteria, consistent with our previous study (6), were: history of elevated serum calcium $(>10.5 \mathrm{mg} \%)$; vitamin D supplementation with a dose of $600 \mathrm{IU} /$ day or more; direct exposure to UV during the past month for greater than eight hours; any kind of malabsorption; history of chronic or acute renal or hepatic disease; current antiseizure medications or glucocorticoids; pregnant/lactating women; and reluctance to consent to the study.

Study visits and sample collection. All participants visited in the GCRU (a unit for clinical research at Boston University Medical Campus). At baseline and every eight weeks, blood was drawn from each subject to determine serum calcium, albumin, creatinine, PTH and total 25(OH)D concentrations. At baseline and after 24 weeks, additional blood and urine were obtained for metabolomics analysis. Quest Diagnostics performed the 25(OH)D and PTH assays as previously described (7). The other blood biochemical tests were performed by Boston Medical Center Clinical Laboratory.

Expression analysis. Genetic expression analysis of circulating mononuclear leukocytes was performed before and after the treatment period to assess differentially expressed genes (DEGs) as previously described (7). RNA extraction was performed using Quiagen's RNeasy kit as described in the manual (Qiagen, Valencia, CA, USA). Isolated RNA was stored at $-86^{\circ} \mathrm{C}$ until microarray analysis. Quality and quantity of the isolated RNA was evaluated by Agilent 2100 Bioanalzyer (Agilent Technologies, Palo Alto, CA, USA). All RNA was sent to the Boston University Microarray Resource Facility for analysis. Procedures are described in the GeneChip ${ }^{\circledR}$ Whole Transcript (WT) Sense Target Labeling Assay Manual (Affymetrix, Sana Clara, CA, USA).

Metabolomics analysis. Blood and urine samples were stored at $80^{\circ} \mathrm{C}$ until processed. Metabolites identified \& quantified in serum and urine samples by The Metabolomics Innovation Center (TMIC). Targeted analysis of serum sample was included 83 metabolites of biogenic amines, amino acids, acylcarnitines, phospholipids and sphingolipids. Metabolites identified and quantified in serum by Direct Flow Injection Mass Spectrometry (DI-LC/MS/MS). Targeted analysis of urine sample was mainly water-soluble metabolites, some lipids and organic acids. Metabolites identified $\&$ quantified in urine samples by GC-MS (TMIC00R4).

Safety and compliance. The amount of vitamin $\mathrm{D}_{3}$ that given was within the guidelines recommended by the Institute of Medicine (now National Academy of Medicine) and the Endocrine Society Guidelines $(13,14)$. Serum calcium, albumin and creatinine was determined every 8 weeks to evaluate for potential toxicity. The participation of an individual who discovered to have serum calcium level above $10.5 \mathrm{mg} / \mathrm{dl}$ or serum creatinine increased by $>20 \%$ was immediately discontinued and the primary care physician was informed (8). 
Table I. Characteristics of the participants by dose group.

\begin{tabular}{lccc}
\hline & \multicolumn{3}{c}{ Vitamin $\mathrm{D}_{3}$ dose assignment (IU/day) } \\
\cline { 2 - 4 } Characteristics & $\begin{array}{c}600 \mathrm{IU} / \text { day } \\
(\mathrm{n}=9)\end{array}$ & $\begin{array}{c}4,000 \text { IU/day } \\
(\mathrm{n}=13)\end{array}$ & $\begin{array}{c}10,000 \text { IU/day } \\
(\mathrm{n}=8)\end{array}$ \\
\hline Gender (female) & 6 & 8 & 5 \\
Race (non-White) & 6 & 5 & 4 \\
Age (years) & $26.3 \pm 2$ & $25.3 \pm 2.1$ & $26.1 \pm 2$ \\
\hline
\end{tabular}

A deidentified bottle containing 60 capsules of vitamin $\mathrm{D}_{3}$ was given to each participant that had one of the doses of vitamin $\mathrm{D}_{3}$ (600 IU/day, 4,000 IU/day or 10,000 IU/day). The bottles were returned at each visit when the vitamin $\mathrm{D}_{3}$ capsules were counted to track compliance as previously described (7).

Statistical and functional analysis. The genomics and metabolomics data were normalized and the quality control and similarity were checked by using Principal Component Analysis (PCA) method as described previously. To compare the metabolomics alteration between groups, a 2-way ANOVA in the linear model was applied (8). The multivariate data matrix was analyzed and the univariate analysis was performed with SPSS software for Mac (SPSS v.25, US). The data were mean centered and Pareto-scaled prior to principal component analysis (PCA) and orthogonal projection to latent structures discriminate analysis (OPLS-DA). Potential markers of interest were extracted from the combining variable importance projection (VIP) plot that was constructed from the loading plots of OPLS-DA. VIP threshold of 1.5 was considered to select the metabolites. In the nonparametric univariate method, the MannWhitney-Wilcoxon test was applied to measure the significance of each metabolite, with results adjusted for multiple testing using false discovery rate (FDR) correction (8). The Pearson correlation was used to study the relations between biomarkers measured and the phenotypic characteristics. Statistical analyses were performed using SPSS software for Mac (SPSS v.25, USA). A $p$-value threshold of 0.05 and also a False Discovery Rate (FDR) of 0.1 considered as significant results. Data are presented as the mean and SD.

Pathways analysis. Pathway enrichment analysis was performed as previously described. DEGs with a significant fold change ( $>2.0$-fold) were selected and reviewed for known associations to pathways involved in cell growth and proliferation using DAVID Bioinformatics 6.8 as previously described (7). Metabolite Set Enrichment Analysis and Pathway Analysis were carried out using the pathway analysis module (MetPA) $(15,16)$ of Metaboanalyst 3.0. Hypergeometric test and relative betweenness centrality $(15,16)$ were used for overrepresentation analysis and pathway topology analysis, respectively.

\section{Results}

A total of 33 adults who met the inclusion/exclusion criteria were enrolled and randomized into each study arm. Thirty participants (90\%) completed the 6-month trial (600 IU/day, $\mathrm{n}=9 ; 4,000$ IU/day, $\mathrm{n}=13 ; 10,000 \quad \mathrm{IU} /$ day, $\mathrm{n}=8)$. The characteristics of participants are shown in Table I.
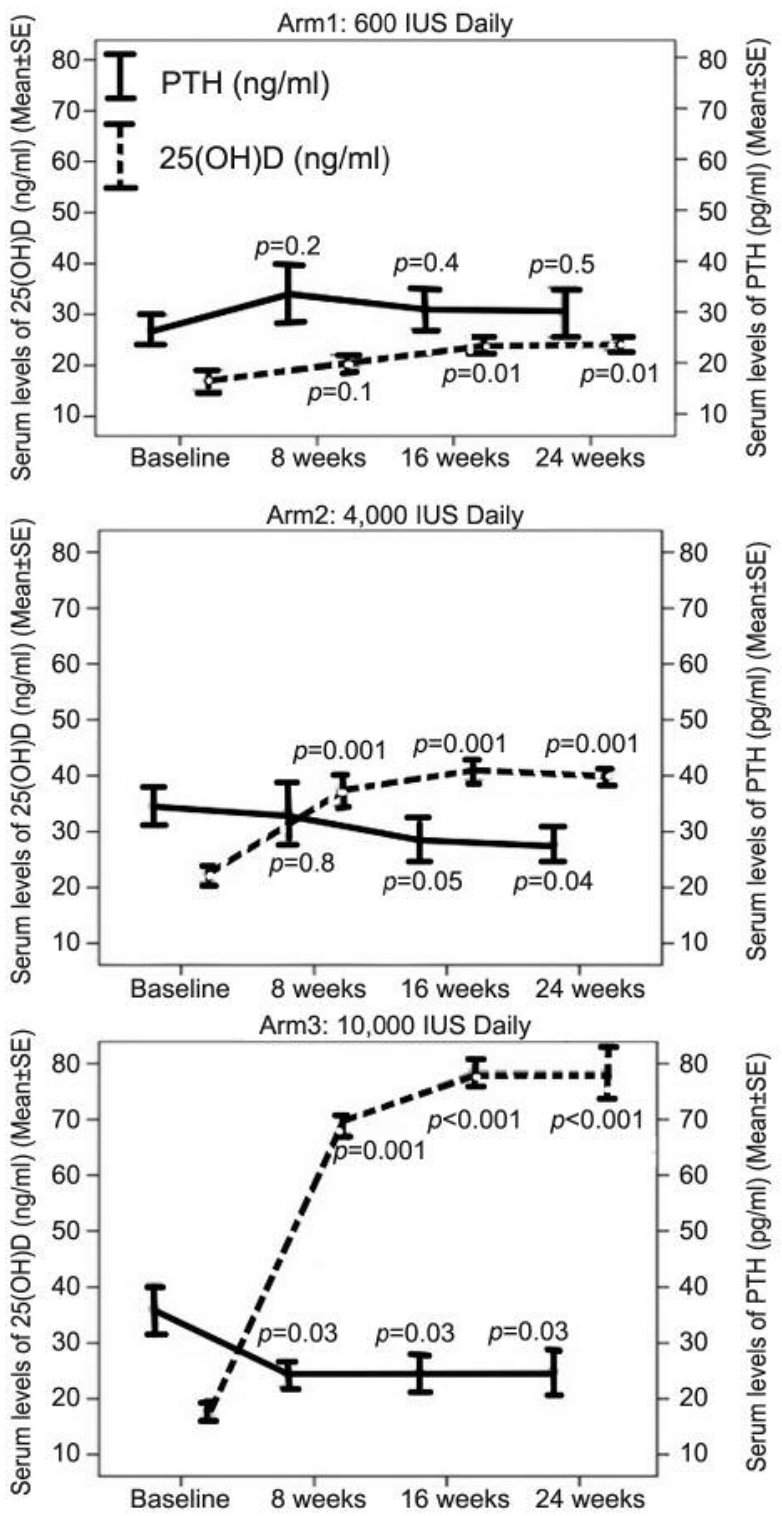

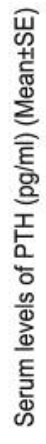

Figure 1. Total concentration of $\mathrm{PTH}$ and $25(\mathrm{OH}) \mathrm{D}$ during vitamin $\mathrm{D}_{3}$ supplementation at baseline and every eight weeks for 24 weeks. This figure is reproduced with permission (8).

The average increase in $25(\mathrm{OH}) \mathrm{D}$ over 24 weeks was 7 $\mathrm{ng} / \mathrm{ml}(18 \mathrm{nmol} / \mathrm{l}), 18 \mathrm{ng} / \mathrm{ml}(45 \mathrm{nmol} / \mathrm{l})$ and $61 \mathrm{ng} / \mathrm{ml}(153$ $\mathrm{nmol} / \mathrm{l}$ ) for $600 \mathrm{IU} /$ day, $4000 \mathrm{IU} /$ day and 10,000 IU/day, respectively. Figure 1 depicts serum concentrations of $25(\mathrm{OH}) \mathrm{D}$ and PTH by dose group. There was no significant change in serum concentrations of PTH or calcium in the $600 \mathrm{IU} /$ day group $(p>0.05)$. All participants in the 4000 and $10,000 \mathrm{IU} /$ day groups achieved $25(\mathrm{OH}) \mathrm{D}$ levels $>30 \mathrm{ng} / \mathrm{ml}$ (>75 nmol/l). There was no significant change in serum calcium for either group. Significant decreases in PTH 
levels of $17.5 \%$ and $33.3 \%$ at 16 weeks were found for the 4000 and 10,000 IU/day group, respectively $(p=0.04)$. PTH levels remained at that level for the remaining 8 weeks (Figure 1, reproduced with permission) (8). There were no significant differences between men and women with respect to changes in serum concentration of calcium, $25(\mathrm{OH}) \mathrm{D}$ or $\mathrm{PTH}$ in response to supplementation with vitamin $\mathrm{D}(8)$.

Differential expression analysis identified a dose-dependent $25(\mathrm{OH}) \mathrm{D}$ alteration in broad gene expression with 162 (86 upregulated, 76 down-regulated), 320 (188 up-regulated, 132 down-regulated), and 1289 (800 up-regulated, 489 downregulated) genes up- or down-regulated in subjects who received 600, 4,000, and 10,000 IU/day dose, respectively (8).

An evaluation of genome wide expression of genes influenced by vitamin $D_{3}$ revealed a greater change in genetic expression in subjects who received 10,000 IU/day/6 months compared to the other two groups. Visualizing of the gene expression alterations in the group of $10,000 \mathrm{IU} /$ day is shown in Figure 2 reproduced with permission (8).

Variable pattern of broad gene expression in response to supplementation with Vitamin $D_{3}$. We compared gene expression between dose groups and related these data to changes in circulating levels of $25(\mathrm{OH}) \mathrm{D}$ and PTH to provide a clearer understanding of the biologic responsiveness to different doses of vitamin D. The pattern of gene expression in response to vitamin D supplementation showed an interindividual variation. Approximately $30 \%$ of the adults who received different doses of vitamin $D_{3}$ supplement demonstrated a much smaller genomic response (8). The serum $25(\mathrm{OH}) \mathrm{D}$ levels of these subjects after 6 months of vitamin $\mathrm{D}_{3}$ supplementation raised to the same degree as the other $70 \%$ (8). This variable pattern of expression is shown in Figure 2, which displays three subjects (ID: 34,46 and 68) that had a very strong genomic response to vitamin $\mathrm{D}_{3}$ supplementation with many genes being up or down regulated, in comparison with one subject (ID: 73) with a moderate response and two subjects (ID: 56 and 66) with a weak response (8).

Our broad gene expression analysis showed that in subjects with a lower genomic response to vitamin $\mathrm{D}_{3}$ supplementation there was a response in $\sim 2-5 \%$ of the genome while in more responsive subjects $>5 \%$ of the genome responded to vitamin $\mathrm{D}_{3}(8)$.

Pathway and functional analysis of the differentially expressed genes. We mapped upregulated genes to the STRING database (7) and selected high confidence for interactions score. The result was significant for proteinprotein interaction $(\mathrm{PPI})$ enrichment $\left(p\right.$-value $\left.=9.33 \mathrm{e}^{-14}\right)$. The key genes in these clusters were HISTIH2B, JUN, NFKB, $T N F, I L 8, H S P A 8, E I F 4 A$ and PRS. This network includes 4 clusters of which one is related to histone modification $(7$,
8). Epigenetic modifications such as histone modification and chromatin regulation are necessary mechanisms to control of gene expression (8). Gene Ontology showed that the genes in this network may regulate DNA accessibility and stability of chromosomes via histone modification as well as remodeling of the nucleosomes. This finding may explain the role of vitamin D supplementation on chromatin accessibility (8). Furthermore, the accessibility of chromatin and vitamin $\mathrm{D}$ supplementation might be considered as factors involved in the pattern of individual alterations in broad gene expression. The other clusters in this network are related to signaling pathways of $N F$-kappa $B, T N F$, NODlike receptor, T cell receptor, $m T O R$, Chemokine, MAPK, Toll-like receptor and pathways in cancer. PPI network was also constructed from the differentially downregulated genes. The key genes in this network are TLR1, CD180 and LRRN3.

Genes for known cancer-related pathways were significantly regulated in the $10,000 \mathrm{IU} /$ day cohort, but not in the 600 or $4,000 \mathrm{IU} /$ day cohorts. In the $10,000 \mathrm{IU} /$ day cohort, 104 genes $(8.1 \% ; p<0.001)$ are involved in a cancerrelated pathway, of which 41 genes $(39.4 \%$; $p<0.0001$; fold change $>2.0 ;$ FDR $<0.01)$ are related to B cell lymphomas. Vitamin $\mathrm{D}_{3}$ significantly $(p<0.05$, FDR $<0.01)$ downregulated expression of the following genes (fold-change): MYD88 (-4.7), HCK (-4.65), SYK (-4.98), BCL2 (-3.99), IKBKB (-4.31), IL6R (-5.31), TLR4 (-5.98), NOTCH1 (-4.32), and MYC (-5.65). Vitamin $\mathrm{D}_{3}$ also significantly upregulated expression of the following genes (fold-change): KDM6A (+4.98), NFKBIZ (+8.97), and NFKBIB (+6.31).

In the $10,000 \mathrm{IU} /$ day cohort, 65 genes are involved in the immune system. The 65 differentially expressed upregulated genes were mapped to the STRING database and screened for significant interactions of the highest confidence (Figure 3).

These biological connections compose 5 clusters and demonstrate that vitamin $\mathrm{D}_{3}$ influences specific functions of the immune system. The key genes within the 5 clusters are phosphatidylinositol 3-kinase catalytic subunit alpha $(P I K 3 C A)$, tumor necrosis factor $(T N F)$, ubiquitin $\mathrm{C}(U B C)$, histone cluster $1 \mathrm{H} 2 \mathrm{~B}(H I S T 1 H 2 B)$, AP-1 transcription factor subunit $(J U N)$, and NF-kB subunits (NFKB and RELA). Additionally, 5 genes were found to have no interactions (PMAIP1, POLB, PPP3CC, SNW1, CREB3). All clusters have biological functions relating to the regulation of immune system processes, response to stress, response to cytokine, cytokine-mediated signaling, cellular response to cytokine stimulus, and response to organic substance, among other immune functions. The significant pathways involved include NF-kB (light blue cluster) and TNF-signaling (upper right-hand dark blue cluster).

The light and dark blue clusters are related to rheumatoid arthritis, atherosclerosis, giant cell arteritis, sarcoidosis, graves' disease, celiac disease, inflammatory bowel disease, 


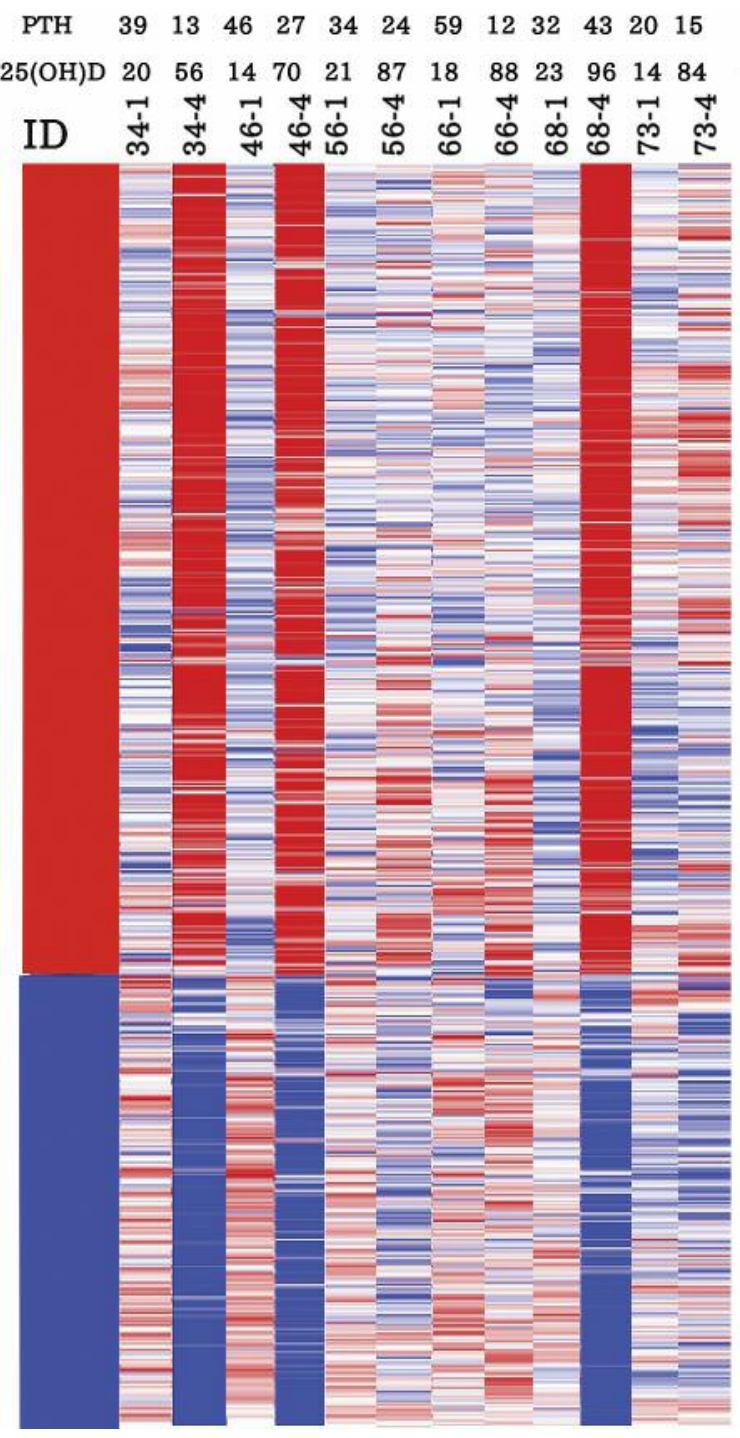

Figure 2. Visualization the gene expression alterations in response to 10,000 IU/day vitamin $D_{3}$ supplementation. The gene-expression alterations are demonstrated by different colors. Upregulation and downregulation of gene expression after 6-months vitamin $D_{3}$ supplementation showed by red and blue, respectively. Trends of gene expression are seen by range of colors from light blue to dark red. The red, white and blue represented of high, average and low gene expression, respectively. This figure is reproduced with permission (8).

and psoriasis. The dark blue cluster alone is related to graves' ophthalmopathy. The red cluster is related to atherosclerosis, sarcoidosis, graves' disease, celiac disease, and psoriasis. The green cluster is related to rheumatoid arthritis, atherosclerosis, celiac disease, and psoriasis. Additionally, the region circled in red corresponds to histone cluster $1 \mathrm{H} 2 \mathrm{~A}, \mathrm{H} 2 \mathrm{~B}, \mathrm{H} 3$, and $\mathrm{H} 4$ family members which are involved in epigenetic modification and systemic lupus erythematosus.

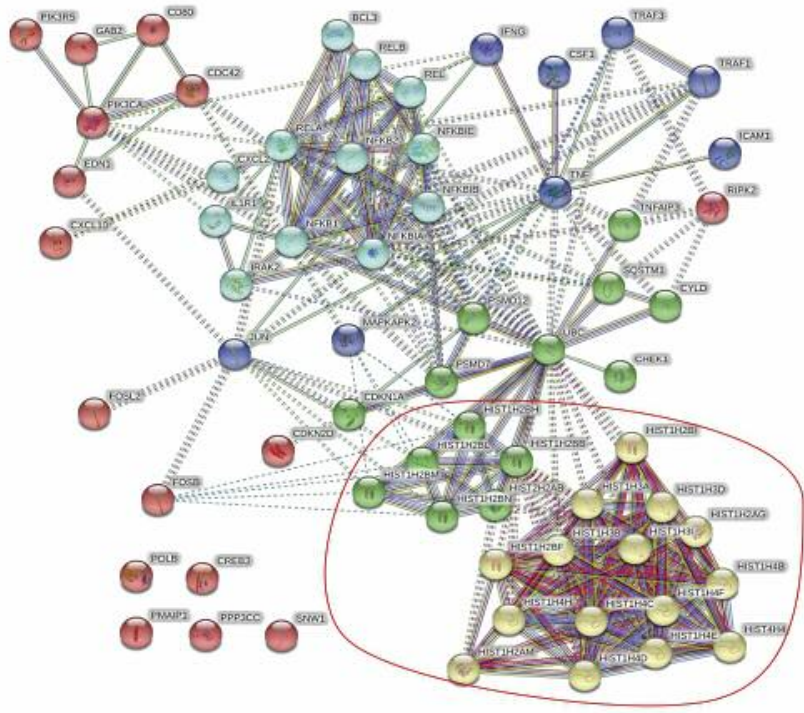

Figure 3. Protein-Protein Interaction (PPI) Network was constructed from differentially upregulated genes contributing to immune systemrelated pathways after 6-month supplementation with vitamin $D_{3}$.

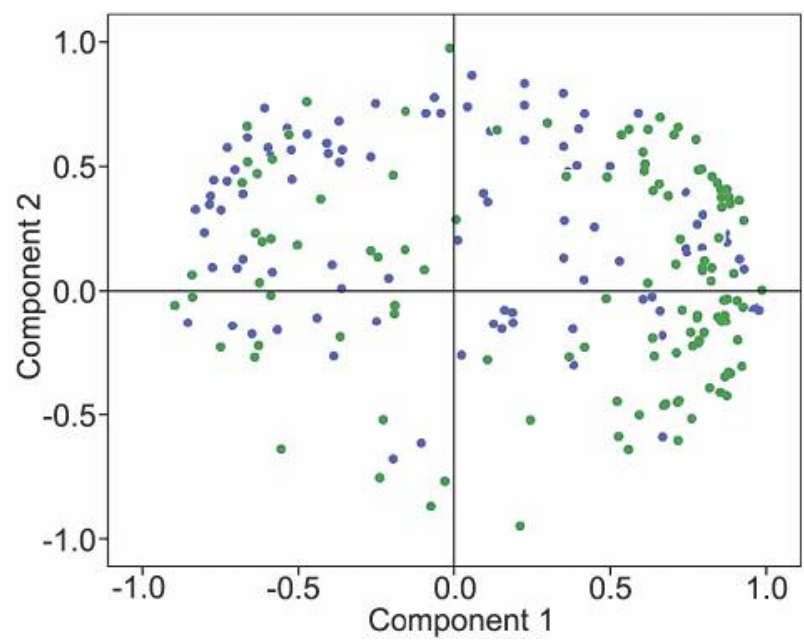

Figure 4. The PCA score plot of the study groups. There was not any pattern of separation in the differential metabolites between 600 IU/day (blue dots) and 10,000 IU/day groups (green dots).

Vitamin $D_{3}$ supplementation and metabolomic profile. Targeted analysis of serum samples was included 83 metabolites of biogenic amines, amino acids, acylcarnitines, phospholipids and sphingolipids. Metabolites were identified and quantified in serum by Direct Flow Injection Mass Spectrometry (DI-LC/MS/MS). Targeted analysis of urine samples was included 36 metabolites of mainly water-soluble metabolites, some lipids and organic acids. Metabolites were identified and quantified in urine samples by GC-MS. 
Table II. The most differentially regulated metabolites by vitamin $D_{3}$ after 6 months supplementation.

\begin{tabular}{lcccc}
\hline Metabolites & Source & $\begin{array}{c}\text { Fold } \\
\text { change }\end{array}$ & $\begin{array}{c}\text { Standard } \\
\text { error }\end{array}$ & $p$-Value \\
\hline Methionine sulfoxide & Urine & 3.7 & 1.0 & 0.001 \\
3 Hydroxyisovaleric acid & Urine & 1.6 & 0.5 & 0.02 \\
3 Methylglutaconic acid & Urine & 1.7 & 0.3 & 0.001 \\
2 Furoylglycine & Urine & 3.2 & 0.8 & 0.05 \\
PC aa C32:2 (Phospholipids) & Serum & 1.5 & 0.1 & 0.005 \\
C0 (Carnitine) & Serum & 1.5 & 0.1 & 0.001 \\
Uracil & Serum & 1.5 & 0.2 & 0.001 \\
C3 (Propionylcarnitine) & Serum & 1.5 & 0.1 & 0.02 \\
C4 (Butyrylcarnitine) & Serum & 1.5 & 0.1 & 0.001 \\
C5 (Valerylcarnitine) & Serum & 1.5 & 0.1 & 0.006 \\
Spermidine & Serum & 1.5 & 0.03 & 0.001 \\
\hline
\end{tabular}

To individually evaluate each metabolite, we calculated the statistically significant ( $p<0.05$ and fold change $>1.5)$ changes of 11 metabolites ( 7 from serum and 4 from urine) after 6 months vitamin $\mathrm{D}_{3}$ supplementation, as shown in Table II.

The enrichment pathway analysis showed that these metabolites mostly involved in the oxidation of branched chain fatty acids. In the principal component analysis (PCA) score plot, the samples of the baseline were only partially separated from the samples of 6 months supplementation with vitamin $\mathrm{D}_{3}$. PCA was first performed to discover intrinsic supplementation-related clusters within the datasets. Following this, partial least-squares discriminant analysis (PLS-DA) and orthogonal partial least-squares discriminant analysis (OPLS-DA) were used to improve separation among the groups and screen differential metabolites. We also didn't observe any reasonable separation of the study groups in the PCA score plot (Figure 4).

There were no significant differences between the 3 study groups $(600,4000$ and 10,000 IU/day) with respect to changes in metabolomic profile in response to supplementation with vitamin $\mathrm{D}_{3}$ as shown by ANOVA.

Following this, partial least-squares discriminant analysis (PLS-DA) and orthogonal partial least-squares discriminant analysis (OPLS-DA) were also used to improve separation among the vitamin D more responsive and less responsive groups and screen differential metabolites. The score plot resulted in unambiguous inter-group separation (Figure 5).

We observed a clear separation between the vitamin $D_{3}$ more responsive and less responsive groups, as shown in the score plot (Figure 5). Differential metabolites were selected based on the separation through the score plot (circle in Figure 5). These metabolites included 44 metabolites from serum and 10 metabolites from urine.

The metabolites with the highest VIP-scores and loading factors are shown in Table III.
Metabolomic pathway analysis. The underlying signaling pathways and molecular networks influenced by vitamin $\mathrm{D}_{3}$ supplementation were explored by MetPA. Identified metabolites contributing to the separation of vitamin D more responsive and less responsive groups were imported into MetPA. This analysis of the relationship between the identified metabolites and diseases such as seizure, hartnup, diabetes mellitus, coronary, schizophrenia and stroke, among others, are mainly predicted (Table IV).

\section{Discussion}

There is an ongoing debate on recommendations of daily allowance for vitamin $\mathrm{D}$ as well as the optimal serum concentration to achieve efficacy (17). Our results demonstrated that PTH plateaued when 25(OH)D levels were $\geq 30 \mathrm{ng} / \mathrm{ml}(75 \mathrm{nmol} / \mathrm{l})$ and confirmed previous observations that serum concentrations of PTH continued to decrease and reached a plateau when circulating levels of $25(\mathrm{OH}) \mathrm{D}$ were $>30 \mathrm{ng} / \mathrm{ml}$ (18-20). The increase in vitamin $\mathrm{D}_{3}$ dose from $4000 \mathrm{IU} /$ day to $10,000 \mathrm{IU} /$ day had no significant additional effect on PTH levels (Figure 1). However, the gene expression analysis demonstrated a dose-dependent effect. Even for subjects who took $600 \mathrm{IU} /$ day of vitamin $\mathrm{D}_{3}$ for 24 weeks, a dose that had little effect on PTH levels, the expression of more than 100 genes was significantly affected. These results indicated that even a small increase in vitamin $\mathrm{D}_{3}$ intake of $600 \mathrm{IU} /$ day for 24 weeks exerted significant genomic effects.

These results may help explain the disparity of conclusions regarding the studies that have evaluated the impact of supplementation with vitamin $\mathrm{D}_{3}$ on serum 25(OH)D improvement and clinical outcomes. The individual's response to vitamin $\mathrm{D}$ is explained by the individual's ability to convert vitamin D to its active metabolites and their interaction with their receptors and response elements (5-7).

Similar to our findings, two recent studies (VitDmet and VitDbol trials) have indicated that there are individual differences in response to supplementation with vitamin D $(5,6)$. Based on their conclusion, this response to vitamin D is explain by epigenetic and genetic individual differences $(5,6)$. In agreement, our results indicated that in subjects with a lower genomic response to vitamin $D_{3}$ supplementation there was a response in $\sim 2-5 \%$ of the genome while in more responsive subjects $>5 \%$ of the genome responded to vitamin $\mathrm{D}_{3}$. The most pronounced vitamin $\mathrm{D}$ response occurred in genes involved in genetic regulation. The major upregulated genes were $H I S T 1 H 2 B$, $J U N, N F K B, T N F, I L 8, H S P A 8, E I F 4 A$ and PRS and the major down regulated genes were $T L R 1, C D 180$ and $L R R N 3$. The upregulated genes were involved in 4 main clusters, one of which is related to histone modification. Epigenetic modifications, such as histone modifications and chromatin 


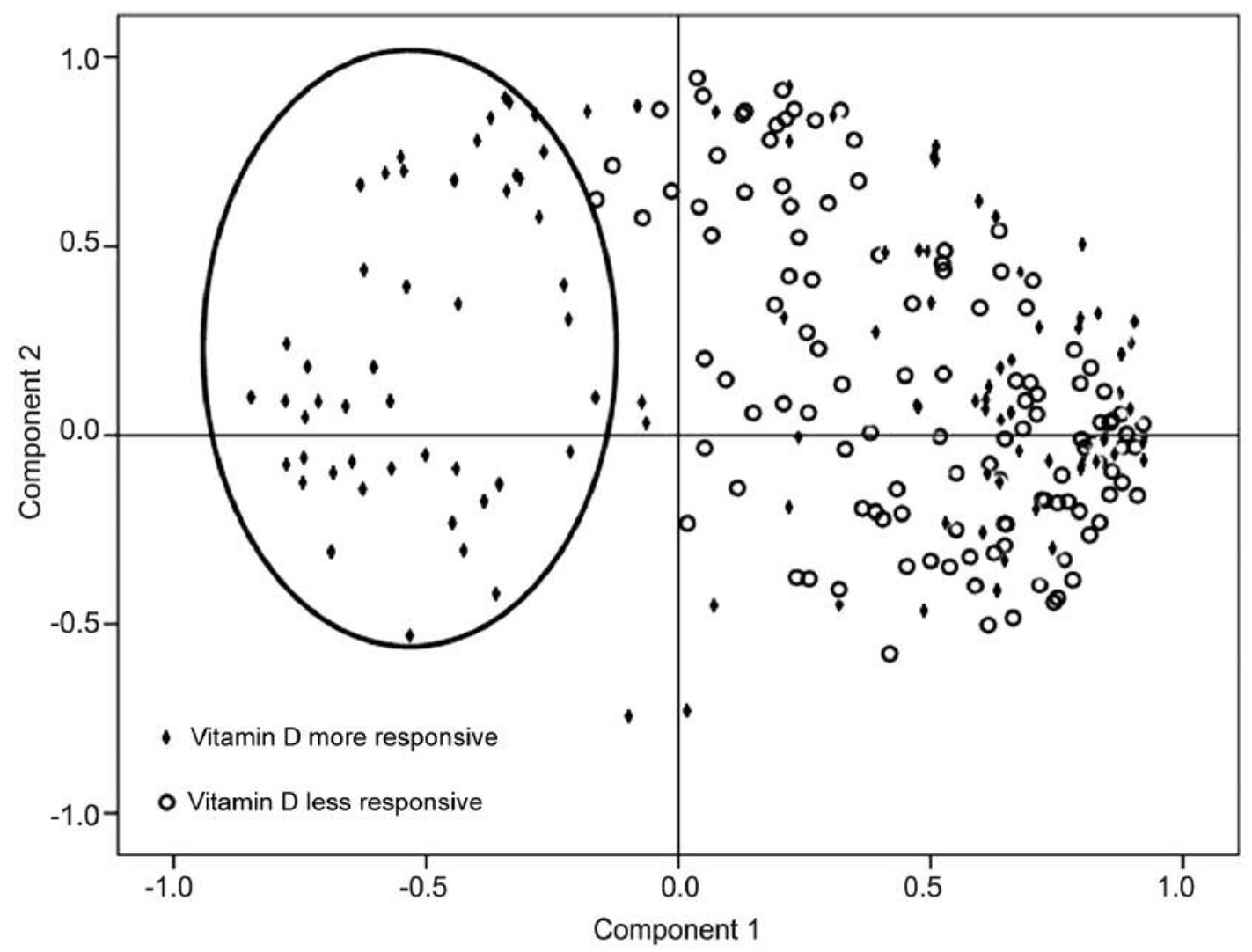

Figure 5. The PCA score plot of the vitamin D in the more responsive and less responsive groups. The score plot resulted in unambiguous intergroup separation. Differential metabolites in the vitamin D more responsive group are shown by diamond dots and in the less responsive by circle dots. The large circle points to the metabolites with the most discriminating power between vitamin D more responsive and less responsive groups.

regulation, are essential parts of gene regulation $(21,22)$. Current findings are consistent with our previous study (7) and several studies $(23,24)$ that showed that vitamin D supplementation resulted in alterations in genes related to epigenetic modifications.

We demonstrated that vitamin D supplementation altered the metabolomic profile by analyzing the serum and urinary metabolomics profile of subjects before and after 6 months supplementation with varying doses of vitamin $D_{3}$. Targeted analysis included 83 metabolites from serum and 36 metabolites from urine. To individually evaluate each metabolite, we calculated the statistically significant $(p<0.05$ and fold change $>1.5$ ) changes of 11 metabolites ( 7 from serum and 4 from urine) after 6 months vitamin $D_{3}$ supplementation, as shown in Table II. These findings are consistent with previous studies implicating vitamin D in metabolomic alteration. The relationship between some of these differentially affected metabolites like spermidine have been previously reported. The previous studies have indicated that spermidine N1-acetyltransferase activity by 1 alpha, $25(\mathrm{OH})_{2} \mathrm{D}_{3}$ is earliest induced metabolite reported to date and that the 1 alpha, $25(\mathrm{OH})_{2} \mathrm{D}_{3}$-induced duodenal synthesis of putrescine occurs by the pathways from both ornithine and spermidine (10). Furthermore, some studies have suggested that both spermidine and spermine are involved in the activation of vitamin D receptor $(9,10)$. Our results showed the ability of metabolomics to identify alterations in the metabolome of subjects more responsive to Vitamin $\mathrm{D}_{3}$ compared with the less responsive subjects. We observed a clear separation in the targeted metabolites between the vitamin $\mathrm{D}_{3}$ more responsive and less responsive groups, as shown in the score plot (Figure 5). Differential metabolites were selected based on the separation through the score plot (red circle in Figure 5). These metabolites included 44 metabolites from serum and 10 metabolites from urine.

Understanding the inter-relationships among genes, gene products and dietary habits is fundamental to identifying those who will benefit most from intervention strategies. Unravelling the multitude of nutrigenomic and metabolomic patterns that arise from the ingestion of foods or their bioactive components provides insights into a tailored approach to diet and supplementation (25). Hence, 
Table III. The list of metabolites that were differentially regulated in vitamin $D$ more responsive and vitamin D less responsive groups.

\begin{tabular}{|c|c|c|c|}
\hline Metabolites & HMDB & PubChem & KEGG \\
\hline Azelaic acid & HMDB0000784 & 2266 & $\mathrm{C} 08261$ \\
\hline Suberic acid & HMDB0000893 & 10457 & $\mathrm{C} 08278$ \\
\hline Glutamine & HMDB0000641 & 5961 & $\mathrm{C} 00064$ \\
\hline Kynurenine & HMDB0000684 & 161166 & $\mathrm{C} 00328$ \\
\hline Valine & HMDB0000883 & 6287 & $\mathrm{C} 00183$ \\
\hline Pimelic acid & HMDB0000857 & 385 & $\mathrm{C} 02656$ \\
\hline Leucine & HMDB0000687 & 6106 & $\mathrm{C} 00123$ \\
\hline Tryptophan & HMDB0000929 & 6305 & $\mathrm{C} 00078$ \\
\hline Arginine & HMDB0000517 & 6322 & $\mathrm{C} 00062$ \\
\hline Histidine & HMDB0000177 & 6274 & $\mathrm{C} 00135$ \\
\hline Isoleucine & HMDB0000172 & 6306 & $\mathrm{C} 00407$ \\
\hline Serotonin & HMDB0000259 & 5202 & $\mathrm{C} 00780$ \\
\hline Tiglylglycine & HMDB0000959 & 6441567 & - \\
\hline 4-Deoxythreonic acid & HMDB0002453 & 10964471 & - \\
\hline Methylsuccinic acid & HMDB0001844 & 10349 & $\mathrm{C} 08645$ \\
\hline Ethylmalonic acid & HMDB0000622 & 11756 & - \\
\hline Hydroxypropionic acid & HMDB0000700 & 68152 & $\mathrm{C} 01013$ \\
\hline trans-Aconitic acid & HMDB0000958 & 444212 & $\mathrm{C} 02341$ \\
\hline Adipic acid & HMDB0000448 & 196 & C06104 \\
\hline $\begin{array}{l}\text { p-Hydroxyphenylacetic } \\
\text { acid }\end{array}$ & HMDB0000020 & 127 & $\mathrm{C} 00642$ \\
\hline 3-keto-2-Methylbutyrate & HMDB0029172 & 11966223 & - \\
\hline Vanillylmandelic acid & HMDB0000291 & 736172 & $\mathrm{C} 05584$ \\
\hline $\begin{array}{l}\text { 3-Hydroxyphenylacetic } \\
\text { acid }\end{array}$ & HMDB0000440 & 12122 & C05593 \\
\hline Uracil & HMDB0000300 & 1174 & C00106 \\
\hline Octadecenoylcarnitine & HMDB0013340 & 53481699 & \\
\hline Glycolic acid & HMDB0000115 & 757 & $\mathrm{C} 00160$ \\
\hline $\begin{array}{l}\text { 5-Hydroxyindoleacetic } \\
\text { acid }\end{array}$ & HMDB0000763 & 1826 & $\mathrm{C} 05635$ \\
\hline L-Acetylcarnitine & HMDB0000201 & 1 & $\mathrm{C} 02571$ \\
\hline Quinolinic acid & HMDB0000232 & 1066 & $\mathrm{C} 03722$ \\
\hline Pimelylcarnitine & HMDB0013328 & 53481675 & - \\
\hline Tetradecenoylcarnitine & HMDB0013330 & 53481679 & \\
\hline $\begin{array}{l}\text { Alpha-Hydroxyisobutyric } \\
\text { acid }\end{array}$ & HMDB0000729 & 11671 & - \\
\hline $\begin{array}{l}\text { 3-Hydroxy-2- } \\
\text { methylglutarate }\end{array}$ & HMDB0029169 & 20070700 & \\
\hline Dodecanoylcarnitine & HMDB0002250 & 168381 & - \\
\hline 3-Hydroxymandelic acid & HMDB0000750 & 86957 & - \\
\hline Pyroglutamic acid & HMDB0000267 & 7405 & C01879 \\
\hline Palmitoylcarnitine & HMDB0000222 & 11953816 & $\mathrm{C} 02990$ \\
\hline
\end{tabular}

nutrigenomics is destined within the next decade to identify complex associations between nutrients and the expression of thousands of genes with simultaneous changes in an equivalent number of metabolites (26).

This research program emphasizes the importance of personalized medicine. Vitamin D supplementation at $10,000 \mathrm{IU} /$ day for 6 months was safe, optimally regulated PTH levels and had a pronounced effect on genetic expression of 1200 genes as well as on metabolomic profile. Furthermore, broad gene expression and metabolomic
Table IV. Metabolite set enrichment analysis for the prediction of disease associated metabolites that were differentially regulated between vitamin $D_{3}$ more responsive and less responsive groups.

\begin{tabular}{lll}
\hline Predicted disease & $p$-Value & FDR \\
\hline Acute seizures & 0.00027 & 0.0738 \\
Different seizure disorders & 0.00122 & 0.166 \\
$\begin{array}{l}\text { Hartnup disease } \\
\text { Diabetes mellitus (MODY), } \\
\text { non-insulin-dependent }\end{array}$ & 0.00582 & 0.529 \\
Pyruvate dehydrogenase deficiency (E3) & 0.011 & 0.655 \\
Continuous ambulatory peritoneal & 0.012 & 0.655 \\
$\quad$ dialysis (CAPD) & 0.0223 & 0.954 \\
Maple syrup urine disease & & \\
Short bowel syndrome & 0.0258 & 0.954 \\
$\quad$ under arginine-free diet) & 0.0316 & 0.954 \\
Ornithine transcarbamylase deficiency (OTC) & 0.0349 & 0.954 \\
Refractory localization-related epilepsy & 0.0349 & 0.954 \\
Schizophrenia & 0.0414 & 1.0 \\
Spastic ataxia & 0.0501 & 1.0 \\
Stroke & 0.0501 & 1.0 \\
\hline
\end{tabular}

profile may predict individual response to vitamin $D_{3}$ supplementation.

\section{Conflicts of Interest}

The A.S., T.K., A.S., R.A., N.C., and R.L. declare no competing interests. M.H. is a consultant for Quest Diagnostics Inc., and was on the speaker's Bureau for Abbott Inc. and Hayat Pharmaceutical Industries Company PLC.

\section{Author's Contributions}

Study design: A.S., R.L. and M.H. Data acquisition: A.S., T.K., A.S., R.A., N.C., R.L. and M.H. Analysis: A.S. and M.H. All authors, A.S., T.K., A.S., R.A., N.C., R.L. and M.H., reviewed and edited the manuscript. M.H. is the guarantor of this work, and, as such, had full access to all the data in the study and takes responsibility for the integrity of the data and the accuracy of the data analysis.

\section{References}

1 Charoenngam N, Shirvani A and Holick MF: Vitamin d for skeletal and non-skeletal health: What we should know. J Clin Orthop Trauma 10(6): 1082-1093, 2019. PMID: 31708633. DOI: 10.1016/j.jcot.2019.07.004

2 Hossein-nezhad A and Holick MF: Vitamin d for health: A global perspective. Mayo Clin Proc 88(7): 720-755, 2013. PMID: 23790560. DOI: 10.1016/j.mayocp.2013.05.011

3 Wacker M and Holick MF: Sunlight and vitamin D: A global perspective for health. Dermatoendocrinol 5(1): 51-108, 2013. PMID: 24494042. DOI: 10.4161/derm.24494

4 Charoenngam N, Shirvani A and Holick MF: The ongoing dlemma of vitamin d supplementation for nonskeletal health and 
bone health. Curr Opin Endocrinol Diabetes Obes 26(6): 301305, 2019. PMID: 31644469. DOI: 10.1097/med.0000000000 000508

5 Carlberg $\mathrm{C}$ and Haq A: The concept of the personal vitamin d response index. J Steroid Biochem Mol Biol 175: 12-17, 2018. PMID: 28034764. DOI: 10.1016/j.jsbmb.2016.12.011

6 Carlberg C: Nutrigenomics of vitamin D. Nutrients 11(3): 676, 2019. PMID: 31644469 . DOI: $10.3390 /$ nu11030676

7 Hossein-nezhad A, Spira A and Holick MF: Influence of vitamin $\mathrm{d}$ status and vitamin $\mathrm{d} 3$ supplementation on genome wide expression of white blood cells: A randomized double-blind clinical trial. PLoS One 8(3): e58725, 2013. PMID: 23527013. DOI: 10.1371 journal.pone.0058725

8 Shirvani A, Kalajian T and Holick MF: Disassociation of vitamin d's calcemic activity and non-calcemic genomic activity and individual responsiveness: A randomized controlled double-blind clinical trial. Sci Rep 9(1): 17685, 2019. DOI: 10.1038/s41598019-53864-1

9 Morishima Y, Inaba M, Nishizawa Y, Morii H, Hasuma T, Matsui-Yuasa I and Otani S: The involvement of polyamines in the activation of vitamin $\mathrm{d}$ receptor from porcine intestinal mucosa. Eur J Biochem 219(1-2): 349-356, 1994. PMID: 8307001. DOI: 10.1111/j.1432-1033.1994.tb19946.x

10 Shinki T, Takahashi N, Kadofuku T, Sato T and Suda T: Induction of spermidine $\mathrm{n} 1$-acetyltransferase by 1 alpha,25dihydroxyvitamin $\mathrm{d} 3$ as an early common event in the target tissues of vitamin D. J Biol Chem 260(4): 2185-2190, 1985. PMID: 3838303.

11 Stepien M, Nugent AP and Brennan L: Metabolic profiling of human peripheral blood mononuclear cells: Influence of vitamin d status and gender. Metabolites 4(2): 248-259, 2014. PMID: 24957025. DOI: $10.3390 /$ metabo4020248

12 Pilz S, Zittermann A, Trummer C, Theiler-Schwetz V, Lerchbaum E, Keppel MH, Grubler MR, Marz W and Pandis M: Vitamin D testing and treatment: A narrative review of current evidence. Endocr Connect 8(2): R27-R43, 2019. PMID: 30650061. DOI: $10.1530 / \mathrm{ec}-18-0432$

13 Holick MF, Binkley NC, Bischoff-Ferrari HA, Gordon CM, Hanley DA, Heaney RP, Murad MH and Weaver CM: Evaluation, treatment, and prevention of vitamin D deficiency: An endocrine society clinical practice guideline. J Clin Endocrinol Metab 96(7): 1911-1930, 2011. PMID: 21646368. DOI: $10.1210 /$ jc.2011-0385

14 Institute of Medicine Committee to Review Dietary Reference Intakes for Vitamin D and Calcium: The National Academies collection: Reports funded by national institutes of health. In: Dietary reference intakes for calcium and vitamin d. Ross AC, Taylor CL, Yaktine AL and Del Valle HB (eds.). National Academies Press (US)National Academy of Sciences. Washington (DC), 2011.

15 Chong J, Wishart DS and Xia J: Using metaboanalyst 4.0 for comprehensive and integrative metabolomics data analysis. Curr Protoc Bioinformatics 68(1): e86, 2019. PMID: 31756036. DOI: $10.1002 / \mathrm{cpbi} .86$
16 Chong J, Yamamoto M and Xia J: Metaboanalystr 2.0: From raw spectra to biological insights. Metabolites 9(3), 2019. PMID: 31756036. DOI: 10.3390/metabo9030057

17 Dalle Carbonare L, Valenti MT, Del Forno F, Caneva E and Pietrobelli A: Vitamin D: Daily vs. Monthly use in children and elderly-what is going on? Nutrients 9(7), 2017. PMID: 28672793. DOI: $10.3390 /$ nu9070652

18 Chapuy MC, Arlot ME, Duboeuf F, Brun J, Crouzet B, Arnaud S, Delmas PD and Meunier PJ: Vitamin D3 and calcium to prevent hip fractures in elderly women. N Engl J Med 327(23): 1637-1642, 1992. PMID: 1331788. DOI: 10.1056/NEJM 199212033272305

19 Holick MF, Siris ES, Binkley N, Beard MK, Khan A, Katzer JT, Petruschke RA, Chen E and de Papp AE: Prevalence of vitamin $\mathrm{d}$ inadequacy among postmenopausal north american women receiving osteoporosis therapy. J Clin Endocrinol Metab 90(6): 3215-3224, 2005. PMID:15797954. DOI: 10.1210/jc.2004-2364

20 Thomas MK, Lloyd-Jones DM, Thadhani RI, Shaw AC, Deraska DJ, Kitch BT, Vamvakas EC, Dick IM, Prince RL and Finkelstein JS: Hypovitaminosis d in medical inpatients. N Engl J Med 338(12): 777-783, 1998. PMID: 9504937. DOI: 10.1056/ nejm199803193381201

21 Fenley AT, Anandakrishnan R, Kidane YH and Onufriev AV: Modulation of nucleosomal DNA accessibility via chargealtering post-translational modifications in histone core. Epigenetics Chromatin 11(1): 11, 2018. PMID: 29548294. DOI: 10.1186/s13072-018-0181-5

22 Zhang G and Pradhan S: Mammalian epigenetic mechanisms. IUBMB Life 66(4): 240-256, 2014. PMID: 24706538. DOI: 10.1002/iub.1264

23 Hossein-nezhad A and Holick MF: Optimize dietary intake of vitamin d: An epigenetic perspective. Curr Opin Clin Nutr Metab Care 15(6): 567-579, 2012. PMID: 23075936. DOI: 10.1097/MCO.0b013e3283594978

24 Wei R, Dhawan P, Baiocchi RA, Kim KY and Christakos S: Pu.1 and epigenetic signals modulate 1,25-dihydroxyvitamin $\mathrm{d} 3$ and c/ebpalpha regulation of the human cathelicidin antimicrobial peptide gene in lung epithelial cells. J Cell Physiol 234(7): 10345-10359, 2019. PMID: 30387140. DOI: $10.1002 /$ jcp.27702

25 Trujillo E, Davis C and Milner J: Nutrigenomics, proteomics, metabolomics, and the practice of dietetics. J Am Diet Assoc 106(3): 403-413, 2006. PMID: 16503231. DOI: 10.1016/ j.jada.2005.12.002

26 Zeisel SH: Nutrigenomics and metabolomics will change clinical nutrition and public health practice: Insights from studies on dietary requirements for choline. Am J Clin Nutr 86(3): 542-548, 2007. PMID: 17823415. DOI: 10.1093/ajcn/86.3.542

Received November 21, 2019

Revised November 27, 2019

Accepted December 2, 2019 